\title{
TOTALLY BOUNDED SETS OF PRECOMPACT LINEAR OPERATORS
}

\author{
THEODORE W. PALMER ${ }^{1}$
}

1. Introduction. In this paper several characterizations of totally bounded sets of precompact operators are given. These lead to an affirmative solution of the conjecture that a collectively compact set $\Omega$ is totally bounded iff $\Omega^{*}=\left\{K^{*}: K \in \Omega\right\}$ is collectively compact.

Let $\mathfrak{X}$ and $\mathfrak{Y}$ be real or complex normed linear spaces with adjoints $\mathfrak{X}^{*}$ and $\mathfrak{Y}^{*}$, respectively. Let the closed unit ball in any of these spaces be denoted by attaching a subscript 1 , and let $[\mathfrak{X}, \mathfrak{Y}]$ be the set of bounded linear operators with domain $\mathfrak{X}$ and range in $\mathfrak{Y}$. An operator $K \in[\mathfrak{X}, \mathfrak{Y}]$ is called precompact [compact] iff $K \mathfrak{X}_{1}=\left\{K x: x \in \mathfrak{X}_{1}\right\}$ is totally bounded [has a compact closure in the norm topology]. If $\mathfrak{Y}$ is complete (i.e., a Banach space), then an operator in $[\mathfrak{X}, \mathfrak{Y}]$ is precompact iff it is compact, and a subset $\Omega$ of $[\mathfrak{X}, \mathfrak{Y}]$ is totally bounded iff its closure is compact.

A subset $\Omega$ of $[\mathfrak{X}, \mathfrak{Y}]$ is called collectively compact iff $\Omega \mathfrak{X}_{1}$ $=\left\{K x: K \in \Omega ; x \in \mathfrak{X}_{1}\right\}$ has compact closure. There is a detailed theory [3] of strongly convergent sequences in a collectively compact set. Characterizing those collectively compact sets which are totally bounded is thus of interest since a strongly (or weakly) convergent sequence converges in norm iff it is contained in a totally bounded set. Investigation of this problem suggested the statements of Theorems 2.1 and 2.2 .

For a complex Hilbert space $\mathfrak{S}$ it was shown in [2] that a collectively compact subset $\Re$ of $[\mathfrak{S}, \mathfrak{S}]$ is totally bounded iff $\Re^{*}$ is collectively compact. The proof used the decomposition of an operator into its self-adjoint and skew-adjoint parts, and spectral theory. In [1] it was shown, by an explicit representation of the operators as tensor products, that a subset $\Omega$ of $[\mathfrak{X}, \mathfrak{Y}]$ is totally bounded if both $\Omega$ and $\Omega^{*}$ are collectively compact and $\{\operatorname{dim} K \mathfrak{X}: K \in \Omega\}$ is bounded. This implies that a collectively compact set $\mathfrak{\Omega} \subseteq[\mathfrak{X}, \mathfrak{Y}]$ with $\max \{\operatorname{dim} K \mathfrak{X}: K \in \Re\}<\infty$ is totally bounded iff $\Omega^{*}$ is collectively compact. Theorem 3.1 proves a conjecture, stated in [1], that an arbitrary collectively compact set $\Omega \subseteq[\mathfrak{X}, \mathfrak{Y}]$ is totally bounded iff $\Omega^{*}$ is collectively compact.

Presented to the Society, January 24, 1968 under the title Collectively compact sets which are totally bounded; received by the editors October 13, 1967.

${ }^{1}$ Sponsored by the Mathematics Research Center, United States Army, Madison, Wisconsin, under Contract No.: DA-31-124-ARO-D-462. 
In addition to Theorem $3.1, \S 3$ contains characterizations of sets $\Omega$ with $\Omega^{*}$ collectively compact, and of totally bounded sets of precompact operators, in terms of the existence of subspaces of finite deficiency on which the operators have small norm. These generalize a known characterization of individual compact operators [5, III.2.3].

In $\$ 4$ a concept is introduced which is weaker than collective compactness in incomplete spaces but nevertheless shares most of its properties.

I would like to thank P. M. Anselone for helpful discussions.

\section{Totally bounded sets of precompact operators.}

Theorem 2.1. A subset $\mathfrak{\Omega}$ of $[\mathfrak{X}, \mathfrak{Y}]$ is a totally bounded set of precompact operators iff

(A) $\Omega \mathfrak{X}_{1}$ is totally bounded, and

(B) $\Re^{*} y^{*}$ is totally bounded for each $y^{*} \in \mathfrak{Y}_{1}^{*}$.

Proof. The set $\Omega^{*}$ is totally bounded iff $\Omega$ is totally bounded. Thus (B) holds whenever $\Omega$ is totally bounded. If in addition $\Re$ consists of precompact operators and $K_{1}, K_{2}, \cdots, K_{n}$ is an $\epsilon$-net for $\Re$, then $\bigcup_{j=1}^{n} K_{j} \mathfrak{X}_{1}$ is a totally bounded $\epsilon$-net for $\Omega \mathfrak{X}_{1}$. This proves the necessity of (A) and (B).

If condition (A) holds, then each $K \in \Omega$ is precompact. Furthermore, the elements of $\mathfrak{Y}_{1}^{*}$ define an equicontinuous family of complex valued functions on the compact closure of $\Omega \mathfrak{X}_{1}$ in the completion of $\mathfrak{V}$. Thus $\mathfrak{Y}_{1}^{*}$ restricted to $\Omega \mathfrak{X}_{1}$ is totally bounded.

Assume that $\Omega$ satisfies (B) as well as (A), and let $\epsilon>0$ be given. We shall complete the proof of the theorem by constructing a finite cover for $\Omega$ consisting of sets of diameter at most $\epsilon$. Choose an $\epsilon / 3$ net $y_{1}{ }^{*}, y_{2}{ }^{*}, \cdots, y_{n}{ }^{*}$ for $\mathfrak{Y}_{1}{ }^{*}$ restricted to $\Re \mathfrak{X}_{1}$. For each $k=1,2, \cdots, n$ choose a finite cover $\left\{\Omega_{k j}: j=1,2, \cdots, m_{k}\right\}$ for $\Omega$ such that $\left\{\Omega_{k+1}^{*} y_{k}^{*}: j=1,2, \cdots, m_{k}\right\}$ is a cover for $\Omega^{*} y_{k}{ }^{*}$ consisting of sets of diameter at most $\epsilon / 3$. Let $\mathfrak{F}$ be the finite family $\mathfrak{F}=\left\{\bigcap_{k=1}^{n} \Omega_{k j_{k}}\right\}$ where $\left\langle j_{1}, j_{2}, \cdots, j_{n}\right\rangle$ runs through all ordered $n$-tuples of integers with $1 \leqq j_{k} \leqq m_{k}$ for $k=1,2, \cdots, n$. Then $\mathfrak{F}$ covers $\Re$. Assume $K_{1}, K_{2} \in \bigcap_{k=1}^{n} \Omega_{k j_{k}}, x \in \mathfrak{X}_{1}$, and $y^{*} \in \mathfrak{Y}_{1}{ }^{*}$. Choose $y_{k}{ }^{*}$ so that $y^{*}-y_{k}{ }^{*}$ restricted to $\Re X_{1}$ has norm at most $\epsilon / 3$. Then

$$
\begin{aligned}
\left|y^{*}\left(K_{1}-K_{2}\right) x\right| \leqq & \left|\left(y^{*}-y_{k}^{*}\right) K_{1} x\right|+\left|y_{k}^{*}\left(K_{1} x-K_{2} x\right)\right| \\
& +\left|\left(y_{k}^{*}-y^{*}\right) K_{2} x\right| \leqq \epsilon .
\end{aligned}
$$

Thus the elements of $\mathfrak{F}$ are sets of diameter at most $\epsilon$, and $\Re$ is totally bounded. 
This proof was suggested by an argument used by K. Vala [6] to characterize totally bounded sets of a more general type of precompact operators.

Theorem 2.2. A subset $\Re$ of $[\mathfrak{X}, \mathfrak{Y}]$ is a totally bounded set of precompact operators iff:

(A) $\Re x$ is totally bounded for each $x \in \mathfrak{X}_{1}$, and

(B) $\mathfrak{\Omega}^{*} \mathfrak{Y}_{1}^{*}$ is totally bounded.

Proof. The proof is essentially dual to that given for Theorem 2.1 except that the proposition that an operator $K$ in $[\mathfrak{X}, \mathfrak{Y}]$ is precompact iff $K^{*}$ is compact must be used [5, III.1.11].

These two theorems suggest that a set $\Omega$ of precompact operators in $[\mathfrak{X}, \mathfrak{Y}]$ might be totally bounded if $\Omega_{x}$ and $\Omega^{*} y^{*}$ were totally bounded for each $x \in \mathfrak{X}_{1}$ and $y^{*} \in \mathfrak{Y}_{1} *$. However, this is not the case, as shown by the following simple example.

ExAmple. Let $\mathfrak{X}=\mathfrak{Y}$ be a separable Hilbert space with a fixed orthonormal basis. Let $E_{n}$ be the orthogonal projection onto the subspace spanned by the first $n$ basis vectors. Then each $E_{n}$ is compact since it has finite dimensional range. Also for a fixed $x$ the set $\left\{E_{n} x: n=1,2, \cdots\right\}=\left\{E_{n}^{*} x: n=1,2, \cdots\right\}$ is totally bounded since $E_{n} x$ converges to $x$. However, $\left\|E_{n}-E_{m}\right\|=1$ for $n \neq m$, so the set is not totally hounded. (Obviously, the use of the Hilbert space adjoint here rather that the Banach space adjoint does not affect the argument.)

\section{Collectively compact sets.}

THEOREM 3.1. A collectively compact subset $\Re$ of $[\mathfrak{X}, \mathfrak{Y}]$ is totally bounded iff $\Re^{*} \subseteq\left[\mathfrak{Y}^{*}, \mathfrak{X}^{*}\right]$ is collectively compact.

Proof. If $\Omega^{*}$ is collectively compact, then $\Omega$ is totally bounded by either Theorem 2.1 or Theorem 2.2. If $\Omega$ is totally bounded, then $\Omega^{*} \mathfrak{Y}_{1}^{*} \subseteq \mathfrak{X}^{*}$ is totally bounded by Theorem 2.2 and thus has compact closure since $\mathfrak{X}^{*}$ is complete. Hence $\Omega^{*}$ is collectively compact.

Theorem 3.2. Let $\Re$ be a subset of $[\mathfrak{X}, \mathfrak{Y}]$. Then the following are equivalent.

(A) $\Omega$ is bounded and for each $\epsilon>0$ there exists a subspace $\mathfrak{X}(\epsilon)$ of finite deficiency in $\mathfrak{X}$ such that $\|K \mid \mathfrak{X}(\epsilon)\| \leqq \epsilon$ for all $K \in \Omega$.

(B) $\Omega^{*}$ is collectively compact.

Proof. Assume (A). For arbitrary $\epsilon>0$ we shall construct an $\epsilon$-net for $\mathfrak{\Omega}^{*} \mathfrak{V}_{1}^{*} \subseteq \mathfrak{X}^{*}$. The subspace $\mathfrak{X}^{\prime}=\mathfrak{X}(\epsilon / 12)$ may be assumed closed since the norm of an operator is the same whether restricted to a subspace or its closure. Let $E^{\prime}$ and $E$ be projection 
operators with $\mathfrak{X}^{\prime}=E^{\prime} \mathfrak{X}$ and $I=E^{\prime}+E$. Then $E$ is a compact operator, so we can choose a finite set $x_{1}, x_{2}, \cdots, x_{n} \in \mathfrak{X}_{1}$ such that for any $x \in \mathfrak{X}_{1}$ there is an $x_{i}$ for which $\left\|E\left(x-x_{i}\right)\right\| \leqq 1$. Since $\Omega^{*} \mathfrak{Y}_{1}^{*}$ is an equicontinuous family of functionals on the compact set $E \mathfrak{X}_{1}$ $\cup\left\{x_{1}, x_{2}, \cdots, x_{n}\right\}$, we can choose a finite set $K_{1}^{*} y_{1}^{*}, K_{2}^{*} y_{2}^{*}, \cdots$, $K_{n}^{*} y_{n}^{*} \in \Omega^{*} \mathfrak{Y}_{1}^{*}$ such that for any $K^{*} y^{*} \in \Omega^{*} \mathfrak{Y}_{1}^{*}$ there is a $K_{k}^{*} y_{k}^{*}$ with $\left\|\left(K^{*} y^{*}-K_{k}^{*} y_{k}^{*}\right) x\right\| \leqq \epsilon / 6$ for all $x \in E \mathfrak{X}_{1} \cup\left\{x_{1}, x_{2}, \cdots, x_{n}\right\}$.

Let $K \in \Omega, y^{*} \in \mathfrak{Y}_{1}^{*}, x \in \mathfrak{X}_{1}$ be arbitrary. Choose $x_{i}$ and $K_{k}^{*} y_{k}^{*}$ as indicated above relative to $x$ and $K^{*} y^{*}$. Then

$$
\begin{aligned}
\left|\left(K^{*} y^{*}-K_{k}^{*} y_{k}^{*}\right) x\right| & \left|\left(K^{*} y^{*}-K_{k}^{*} y_{k}^{*}\right) x_{i}\right|+\left|\left(K^{*} y^{*}-K_{k}^{*} y_{k}^{*}\right)\left(x-x_{i}\right)\right| \\
\leqq & \epsilon / 6+\left|y^{*} K E^{\prime}\left(x-x_{i}\right)\right|+\left|y_{k}^{*} K_{k} E^{\prime}\left(x-x_{i}\right)\right| \\
& +\left|\left(K^{*} y^{*}-K_{k}^{*} y_{k}^{*}\right) E\left(x-x_{i}\right)\right| \\
\leqq & \epsilon / 6+2(\epsilon / 12)\left\|E^{\prime}\left(x-x_{i}\right)\right\|+2(\epsilon / 6) \\
\leqq & \epsilon / 6+(\epsilon / 6)\left(\left\|x-x_{i}\right\|+\left\|E\left(x-x_{i}\right)\right\|\right)+\epsilon / 3 \leqq \epsilon .
\end{aligned}
$$

Thus $\Omega^{*}$ is collectively compact since $\mathfrak{X}^{*}$ is complete.

Assume (B). Then $\Omega^{*}$, and hence $\Omega$, is bounded. Choose an $\epsilon$-net $K_{1}^{*} y_{1}^{*}, K_{2}^{*} y_{2}^{*}, \cdots, K_{n}^{*} y_{n}^{*}$ from $\Omega^{*} \mathfrak{Y}_{1}^{*}$. Let $\mathfrak{X}(\epsilon)=\bigcap_{j=1}^{n}$ null space $\left(K_{j}^{*} y_{j}^{*}\right)$. Let $x \in \mathfrak{X}(\epsilon)$ and $K \in \Omega$. Choose $y^{*} \in \mathfrak{V}_{1}^{*}$ such that $y^{*} K x$ $=\|K x\|$, and choose $K_{j}^{*} y_{j}^{*}$ such that $\left\|K^{*} y^{*}-K_{j}^{*} y_{j}^{*}\right\| \leqq \epsilon$. Then $\|K x\|=y^{*} K x=\left(K^{*} y^{*}-K_{j}^{*} y_{j}^{*}\right) x \leqq \epsilon\|x\|$.

Theorem 2.2 and Theorem 3.2 can be used to prove another characterization of totally bounded sets of precompact operators.

TheOREM 3.3. A subset $\Re$ of $[\mathfrak{X}, \mathfrak{Y}]$ is a totally bounded set of precompact operators iff the following conditions hold:

(A) $\Re x$ is totally bounded for each $x \in \mathfrak{X}_{1}$.

(B) For each $\epsilon>0$ there is a subspace $\mathfrak{X}(\epsilon)$ of finite deficiency in $\mathfrak{X}$ which satisfies $\|K \mid \mathfrak{X}(\epsilon)\| \leqq \epsilon$ for each $K \in \Omega$.

Proof. In (B) the subspace $\mathfrak{X}^{\prime}=\mathfrak{X}(1)$ may be assumed closed since the norm of an operator is the same whether restricted to a subspace or to its closure. Thus there is a bounded projection $E^{\prime}$ onto $\mathfrak{X}^{\prime}$. Let $E=I-E^{\prime}$. Since $E \mathfrak{X}$ is finite dimensional and hence complete, the uniform boundedness principle together with (A) implies a bound $b$ for $\{\|K \mid E \mathfrak{X}\|: K \in \Omega\}$. Thus $\sup \{\|K\|: K \in \Omega\} \leqq 1\left\|E^{\prime}\right\|+b\|E\|$. Since (A) and (B) imply that $\Omega$ is bounded, the theorem now follows easily from Theorems 3.2 and 2.2. 
4. Collectively precompact sets. Many of the results obtained for collectively compact sets in (incomplete) normed linear spaces remain valid for the wider class of sets of operators defined below.

DEFINITION 4.1. A subset $\mathfrak{R}$ of $[\mathfrak{X}, \mathfrak{V}]$ is called collectively precompact iff $\Omega \mathfrak{X}_{1}$ is totally bounded.

The terminology is suggested by the standard definition of a precompact operator $K$ as one for which $K \mathfrak{X}_{1}$ is totally bounded. Each operator in a collectively precompact set is precompact. Obviously a collectively compact set $\mathfrak{R} \subseteq[\mathfrak{X}, \mathfrak{V}]$ is always collectively precompact and the converse holds if $\mathfrak{Y}$ is a Banach space.

Theorems 2.1 and 2.2 can now be restated in a concise but slightly weakened form.

Theorem 4.2. A subset $\mathfrak{\Omega}$ of $[\mathfrak{X}, \mathfrak{Y}]$ is a totally bounded set of precompact operators iff $\Omega$ and $\Omega^{*}$ are collectively precompact.

Theorems 3.1 and 3.2 remain valid if "collectively compact" is replaced by "collectively precompact." Furthermore every statement in $\$ 2$ of [2] and $\$ 3$ of [4] remains valid if "collectively compact," "compact operator," and "has compact closure" are replaced by "collectively precompact," "precompact operator" and "is totally bounded," respectively. In certain cases this assertion is most easily checked by passing to $[\mathfrak{X}, \tilde{\mathfrak{Y}}]$ where $\tilde{\mathfrak{Y}}$ is the completion of $\mathfrak{Y}$. In no case is the verification difficult.

We conclude by giving a characterization of collectively precompact sets similar to Theorem 3.2.

TheOREM 4.3. A subset $\Re$ of $[\mathfrak{X}, \mathfrak{Y}]$ is collectively precompact iff it is bounded and for every $\epsilon>0$ there exists a subspace $\mathfrak{V}(\epsilon)$ of finite deficiency in $\mathfrak{Y}^{*}$ such that $\left\|K^{*} \mid \mathfrak{Y}(\epsilon)\right\| \leqq \epsilon$ for all $K \in \Omega$.

Proof. If $\Omega$ is collectively precompact, it is certainly bounded. Choose an $\epsilon$-net $K_{1} x_{1}, K_{2} x_{2}, \cdots, K_{n} x_{n}$ for $\Re \mathfrak{X}_{1}$ and let $\mathfrak{Y}(\epsilon)$ $=\left\{y^{*}: y^{*} K_{j} x_{j}=0, j=1,2, \cdots, n\right\}$. If $y^{*} \in \mathfrak{V}(\epsilon)_{1}$ and $x \in \mathfrak{x}_{1}$, then $\left|K^{*} y^{*} x\right|=\left|y^{*}\left(K x-K_{j} x_{j}\right)\right| \leqq \epsilon$ for suitable $K_{j} x_{j}$. Thus $\left\|K^{*} \mid \mathfrak{V}(\epsilon)\right\| \leqq \epsilon$.

To prove the sufficiency of the condition, note that $\Omega^{*}$ satisfies Theorem 3.2 so $\Re \mathfrak{X}_{1} \subseteq \Omega^{* *} \mathfrak{X}_{1}^{* *}$ is totally bounded.

The reader can easily formulate a theorem which bears the same relation to Theorems 2.1 and 4.3 as Theorem 3.3 bears to Theorems 2.2 and 3.2 .

\section{REFERENCES}

1. P. M. Anselone, Collectively compact and totally bounded sets of linear operators, MRC Tech. Summary Rep. No. 766, J. Math. Mech. 17 (1968), 613-622. 
2. P. M. Anselone, and T. W. Palmer, Collectively compact sets of linear operators, MRC Tech. Summary Rep. No. 740, Pacific J. Math. 25 (1968), 417-422.

3. - Spectral analysis of collectively compact, strongly convergent operator sequences, MRC Tech. Summary Rep. No. 741, Pacific. J. Math. 25 (1968), 423-431.

4. - Spectral properties of collectively compact sets of linear operators, MRC Tech. Summary Rep. No. 767, J. Math. Mech. 17 (1968), 853-860.

5. S. Goldberg, Unbounded linear operators; Theory and applications, McGrawHill, New York, 1966.

6. K. Vala, On compact sets of compact operators, Ann. Acad. Sci. Fenn. Ser. A1 351 (1964).

Mathematics Research Center, Madison, Wisconsin and UNIVERSITY OF KANSAS 